\title{
Palmitate Activates CCL4 Expression in Human Monocytic Cells via TLR4/MyD88 Dependent Activation of NF-kB/MAPK/ PI3K Signaling Systems
}

\author{
Shihab Kochumon ${ }^{\mathrm{a}} \quad$ Ajit Wilson $^{\mathrm{a}} \quad$ Betty Chandy $^{\mathrm{b}}$ Steve Shenouda ${ }^{\mathrm{a}}$ \\ Jaakko Tuomilehtoc Sardar Sindhu ${ }^{\mathrm{d}}$ Rasheed Ahmad ${ }^{\mathrm{a}}$ \\ ammunology Unit, ${ }^{\text {a }}$ National Biobank Core Facility, 'Research Division, ${ }^{\mathrm{d} A n i m a l}$ and Zebrafish Core \\ Facility, Dasman Diabetes Institute, Kuwait
}

\section{Key Words}

Palmitate • CCL4/MIP-1beta • TLR4

\begin{abstract}
Background/Aims: Obesity is associated with adipose tissue inflammation which plays a key role in the development of insulin resistance and type 2 diabetes (T2D). Saturated free fatty acids (SFAs) levels are found to be elevated in obesity and T2D. Chemokines are known to have potent inflammatory functions in a wide range of biological processes linked to immunological disorders. Since CCL4 (Chemokine (C-C motif) ligand 4), also known as macrophage inflammatory protein-1 $\beta$ (MIP-1 $\beta$ ), plays an important role in the migration of monocytes into the adipose tissue, we investigated the expression of CCL4 in monocytic cells/ macrophages following activation with free fatty acid palmitate. Methods: Human monocytic cell line THP-1 and macrophages derived from THP-1 and primary monocytes were stimulated with palmitate and LPS (positive control). CCL4 expression and secretion were measured with real time RT-PCR and ELISA respectively. Signaling pathways were identified by using THP-1-XBlue ${ }^{\mathrm{TM}}$ cells, THP-1-XBlue ${ }^{\mathrm{TM}}$-defMyD cells, anti-TLR4 mAb and TLR4 siRNA. Results: Palmitate induces CCL4 expression at both mRNA and protein levels in human monocytic cells. Palmitate-induced CCL4 production was markedly suppressed by neutralizing anti-TLR-4 antibody. Additionally, silencing of TLR4 by siRNA also significantly suppressed the palmitateinduced up-regulation of CCL4. MyD88-deficient cells did not express CCL4 in response to palmitate treatment. Inhibition of NF-kB and MAPK pathways suppressed the palmitate mediated induction of CCL4. Moreover, induction of CCL4 was blocked by PI3 Kinase inhibitors LY294002 and wortmannin. Conclusion: Collectively, our results show that palmitate induces CCL4 expression via activation of the TLR4-MyD88/NF-kB/MAPK/ PI3K signaling cascade. Thus, our findings suggest that the palmitate-induced CCL4 production might be an underlying mechanism of metabolic inflammation.




\section{Cellular Physiology Cell Physiol Biochem 2018;46:953-964

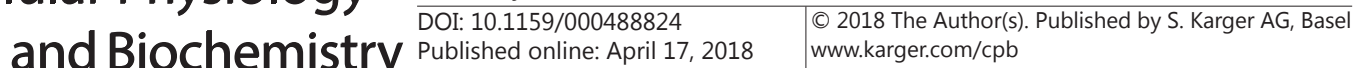

Kochumon et al.: Free Fatty Acid Palmitate Induces CCL4

\section{Introduction}

Macrophage Inflammatory Proteins (MIP) belong to the family of chemotactic cytokines known as chemokines which are divided into two major forms, MIP-1 $\alpha$ (CCL3) and MIP$1 \beta$ (CCL4). MIP- $1 \alpha$ and MIP-1 $\beta$ are important in immune responses to infection and inflammation. CCL3 and CCL4 can lead to acute neutrophilic inflammation via activation of human granulocytes. These two chemokines induce synthesis and release of other proinflammatory cytokines such as interleukin-1 (IL-1), IL- 6 and TNF- $\alpha$ from fibroblasts and macrophages. Regulation of CCL3 production is well defined, however, much less work has been done regarding the production and regulation of CCL4. CCL4 is a crucial chemotactic mediator for monocytes/macrophages (MФ) recruitment [1]. In addition, CCL4 can also attract other immune cells, namely $\mathrm{T}$ lymphocytes, dendritic cells and natural killer (NK) cells [2]. CCL4 production by neutrophils contributes to inflammation by recruiting other leukocytes into the inflamed tissues, leading either to inflammation resolution through efferocytosis by macrophages or to the development of chronic inflammation. CCL4 is associated with the pathogenesis of many diseases including psoriasis vulgaris [3], sarcoidosis [4], cystic fibrosis [5], multiple sclerosis [6] and systemic lupus erythematosus [7]. Chemokines are produced by an array of both immune and nonimmune cells and their secretion is typically induced by pro-inflammatory cytokines such as interleukin (IL)-1, IL-2, TNF- $\alpha$ as well as viruses, bacterial lipopolysaccharide (LPS), and complement fraction (C)5a and leukotriene (LT)B4.

In obesity, CCL4 levels are found to be increased and CCL4 plays a role in metabolic inflammation [8]. Changes in immune system functioning in obesity and T2D lead to the increased production of free fatty acids (FFAs) [9]. Several reports showed a possible mechanistic link between FFAs and innate immune toll-like receptors (TLRs) in metabolic disease. TLRs are a family of pattern recognition receptors that activate the innate immune responses upon interaction with various pathogen-associated molecular patterns, including lipids, lipoproteins, proteins and FFAs [10-12]. After ligand binding, TLRs engage Toll/Interleukin-1 receptor (TIR) domain-containing adaptor proteins (either myeloid differentiation primary-response protein 88 (MyD88) and MyD88-adaptor-like protein (MAL), or TIR domain-containing adaptor protein inducing IFN $\beta$ (TRIF) and TRIF-related adaptor molecule (TRAM). This association triggers the recruitment and activation of IRAK1, forming a complex with TRAF6 and activating transcription factors including NF-kB and AP-1 $[10,13-15]$. FFAs induce inflammatory mediators' production in immune cells via TLR-4. Furthermore, TLR4 signaling is important for high fat diet-induced insulin resistance, presumably by mediating inflammatory responses within adipose tissue and skeletal muscle [11]. Palmitate, which is the most prevalent dietary SFAs, was reported to induce proinflammatory cytokines including IL-6, TNF- $\alpha$, IL-8, and IL-1 $\beta[16,17]$. We have shown recently that palmitate induces the production of MMP-9 in human monocytic cells via TLR4/ MyD88 [10]. However, its role in the induction of CCL4 is not yet clear. We hypothesized that palmitate could trigger the production of CCL4 in monocytic cells/macrophages via activation of TLR4 signaling pathway. Herein, we show that palmitate can induce CCL4 in monocytes and this induction involves TLR4/MyD88/NF-kB/AP-1 axis. Furthermore, we also found that mechanism of TLR4 activation leading to CCL4 production is dependent on PI 3-kinase activity, and is not restricted to the activation of NF-kB/AP-1.

\section{Materials and Methods}

\section{Cell Culture}

Human monocytic leukemia cell line THP-1 was purchased from American Type Culture Collection (ATCC) and grown in RPMI-1640 culture medium (Gibco, Life Technologies, Grand Island, USA) supplemented with 10\% fetal bovine serum (Gibco, Life Technologies, Grand Island, NY, USA), 2 mM glutamine (Gibco, 


\section{Cellular Physiology Cell Physiol Biochem 2018;46:953-964 \begin{tabular}{ll|l} 
DOI: 10.1159/000488824 & O 2018 The Author(s). Published by S. Karger AG, Basel \\
www.karger.com/cpb
\end{tabular}}

Kochumon et al.: Free Fatty Acid Palmitate Induces CCL4

Invitrogen, Grand Island, NY, USA), $1 \mathrm{mM}$ sodium pyruvate, $10 \mathrm{mM}$ HEPES, $100 \mathrm{ug} / \mathrm{ml}$ Normocin, $50 \mathrm{U} /$ $\mathrm{ml}$ penicillin and $50 \mu \mathrm{g} / \mathrm{ml}$ streptomycin (P/S; (Gibco, Invitrogen, Grand Island, NY, USA), and incubation at $37^{\circ} \mathrm{C}$ (with humidity) in $5 \% \mathrm{CO}_{2}$. THP-1-XBlue cells stably expressing a secreted embryonic alkaline phosphatase (SEAP) reporter inducible by NF-KB and AP-1 were purchased from InvivoGen (InvivoGen, San Diego, CA, USA). THP-1-XBlue ${ }^{\mathrm{TM}}$-defMyD cells (cells deficient in MyD88 activity or MyD88-/- THP-1 cells) were purchased from InvivoGen (San Diego, CA, USA). THP-1-XBlue cells were cultured in complete RPMI medium with the addition of zeocin $(200 \mu \mathrm{g} / \mathrm{ml})$ to select for cells expressing the SEAP -NF- $\mathrm{kB} / \mathrm{AP}-1$ reporter (InvivoGen, San Diego, CA, USA). THP-1-XBlue ${ }^{\mathrm{TM}}$-defMyD cells were cultured in complete RPMI medium with the addition of Zeocin $(200 \mathrm{ug} / \mathrm{ml})$ and HygroGold (100 ug/ml) (InvivoGen, San Diego, CA, USA). Prior to stimulation, THP- 1 cells were transferred to normal medium and plated in 12-well plates (Costar, Corning Incorporated, Corning, NY, USA) at $1 \times 10^{6}$ cells/well cell density unless indicated otherwise.

\section{Cell stimulation and macrophage differentiation}

Monocytic cells were plated in 12-well plates (Costar, Corning Incorporated, Corning, NY, USA) at 1 $\times 10^{6}$ cells/well concentration unless indicated otherwise. Cells were stimulated with palmitate $200 \mu \mathrm{M}$ (Sigma, San Diego, CA, USA) or LPS (10 ng/ml; Sigma, Saint Louis, MO, USA) or $0.1 \%$ BSA for $24 \mathrm{hrs}$ at $37^{\circ} \mathrm{C}$. Cells were harvested for RNA isolation and culture media were collected for measuring CCL4/MIP-1 $\beta$ secretion. THP- 1 cells were plated in 12 well plate at $1 \times 10^{6}$ cell $/ \mathrm{ml}$ concentration and treated with PMA $(50 \mathrm{ng} / \mathrm{ml})$ for 72 hours at $37^{\circ} \mathrm{C}$. Differentiated macrophages were then stimulated with palmitate $(200 \mu \mathrm{M})$ or LPS $(10 \mathrm{ng} / \mathrm{ml})$ or $0.1 \%$ BSA for $24 \mathrm{hrs}$ at $37^{\circ} \mathrm{C}$. Cells were harvested for RNA isolation and culture media were collected for measuring CCL4/MIP- $1 \beta$ secretion.

\section{Peripheral blood mononuclear cells (PBMCs) collection and differentiation.}

The human peripheral blood $(40 \mathrm{ml})$ samples were collected from healthy donors in EDTA vacutainer tubes through venipuncture by phlebotomist at the Dasman Diabetes Institute (DDI) and PBMCs were isolated by using Histo-Paque density gradient method as described ([13]. PPBMCs were plated in 6-well plates (Costar, Corning Incorporated, Corning, NY, USA) at $3 \times 10^{6}$ cells/well in starvation medium for 3 hours at $37^{\circ} \mathrm{C}$. Non-adhered cells were removed and macrophages that have adhered to the plate were washed with culture media without serum and incubated for $24 \mathrm{hrs}$ in RPMI with 2\% Fetal Bovine serum. Cells were then stimulated with palmitate (200 $\mu$ M; Sigma, San Diego, CA, USA) or LPS (10 ng/ml; Sigma, Saint Louis, MO, USA) or $0.1 \%$ BSA for $24 \mathrm{hrs}$ at $37^{\circ} \mathrm{C}$. Cells were harvested for RNA isolation and culture media were collected for measuring CCL4/MIP-1 $\beta$ secretion.

\section{TLR4 neutralization}

THP- 1 cells were treated with $2 \mu \mathrm{g} / \mathrm{mL}$ of neutralizing TLR4 mAb (cat\#mab2-htlr4) or isotype-matched control (IgA; Cat\#: maba2-ctrl) for 40 minutes. Antibody-treated cells were stimulated with palmitate or BSA and incubated for $24 \mathrm{hrs}$. Cells and culture media were collected.

\section{Measurement of NF- $\mathrm{KB} / A P-1$ activity}

THP-1-XBlue cells (InvivoGen, San Diego, CA) are THP-1 cells stably transfected with a reporter construct, expressing a secreted embryonic alkaline phosphatase (SEAP) gene under the control of a promoter inducible by the transcription factors NF- $\mathrm{\kappa B}$ and AP-1. Upon stimulation, NF- $\mathrm{\kappa B}$ and AP-1 are activated and subsequently the secretion of SEAP is stimulated. THP- 1 XBlue cells were stimulated with palmitate $(200 \mu \mathrm{M})$ or LPS $\left(10 \mathrm{ng} / \mathrm{ml}\right.$; positive control) for $24 \mathrm{hr}$ at $37^{\circ} \mathrm{C}$. Levels of SEAP were detected in the culture media after $3 \mathrm{hrs}$ incubation of supernatants with Quanti-Blue solution (InvivoGen, San Diego, CA, USA) at $650 \mathrm{~nm}$ wave length by ELISA reader.

\section{Small interfering RNA (siRNA) transfections}

THP-1 cells were washed and resuspended in $100 \mathrm{ul}$ of nucleofector solution provided with the Amaxa Noclecfector Kit $\mathrm{V}$ and transfected separately with siRNA-TLR4 (30nM; OriGene Technologies, Inc. MD, USA), scramble (control) siRNA (30nM; OriGene Technologies, Inc. MD, USA, USA) and pmaxGFP (0.5 ug; Amaxa Noclecfector Kit V for THP-1, Lonza). All transfection experiments were performed with Amaxa Cell Line Nucleofector Kit V for THP-1 (Lonza, Germany) by using Amaxa Electroporation System (Amaxa Inc, Germany) according to the manufacturer's protocol. After $36 \mathrm{hrs}$ transfection, cells were treated with 


\section{Cellular Physiology Cell Physiol Biochem 2018;46:953-964

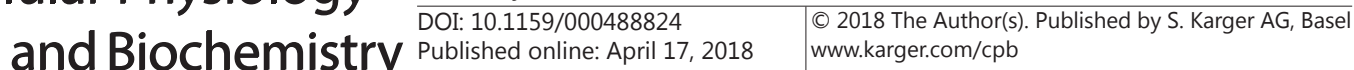

Kochumon et al.: Free Fatty Acid Palmitate Induces CCL4

palmitate $(200 \mu \mathrm{M})$ for $24 \mathrm{hrs}$. Cells were harvested for RNA isolation and culture media were collected for measuring CCL4 secretion. siRNA Transfection efficiency was analyzed by fluorescence microscopy and TLR4 gene Knock down level was assessed by Real Time-PCR using TLR4 gene specific primer probes.

\section{Real Time RT-PCR}

RNeasy Mini Kit (Qiagen, Valencia. CA, USA) was used to extract total RNA. The cDNA was synthesized using $1 \mu \mathrm{g}$ of total RNA using high capacity cDNA reverse transcription kit (Applied Biosystems, Foster city, CA, USA). 50ng cDNA was used in each real time polymerase chain reaction (PCR) reaction. For real time PCR, complementary DNA was amplified with Inventoried TaqMan Gene Expression Assay products (CCL4/ MIP-1 ß: Hs99999148_m1; GAPDH: Hs03929097_g1; TLR-4: Hs00152939_ml) containing two gene-specific primers and one TaqMan MGB probe (6-FAM dye-labeled) using a TaqMan ${ }^{\circledR}$ Gene Expression Master Mix (Applied Biosystems, Foster city, CA, USA) in a 7500 Fast Real-Time PCR System (Applied Biosystems, Foster City, CA, USA). The mRNA levels were normalized against GAPDH mRNA and the amounts of CCL4 mRNA relative to control were calculated with $\Delta \Delta \mathrm{Ct}$-method. Relative mRNA expression was expressed as fold expression over average of control gene expression. The expression level in control treatment was assumed to be 1 .

\section{Secreted CCL4 quantification}

Secreted CCL4 protein in supernatants of monocytic cells stimulated with palmitate or LPS was quantified using sandwich ELISA following the manufacturer's instructions (R\&D systems, Minneapolis, USA).

\section{Western blotting}

THP-1 cells treated with palmitate at different time points were harvested and incubated for $30 \mathrm{~min}$ with lysis buffer (10X Lysis Buffer, Cell signaling, USA). The lysates were then centrifuged at $14000 \mathrm{~g}$ for 10 $\mathrm{min}$ and the supernatants were collected. Protein concentration in the lysates was measured by Quickstart Bradford Dye Reagent, 1x Protein Assay kit (Bio-Rad Laboratories, Inc, CA). Protein (20 $\mu \mathrm{g})$ samples were mixed with sample loading buffer, heated for $5 \mathrm{~min}$ at $95^{\circ} \mathrm{C}$ and resolved by $12 \%$ SDS-PAGE. Cellular proteins were transferred to Immuno-Blot PVDF membrane (Bio-Rad Laboratories, USA) by electro blotting. The membranes were then blocked with $5 \%$ non-fat milk in PBS for $1 \mathrm{~h}$, followed by incubation with primary antibodies against p-ERK1/2, p-JNK, p-c-Jun, p-NF-kB, p-PI3K, ERK1/2, JNK, c-Jun, NF-kB and PI3K in 1:1000 dilution at $4^{\circ} \mathrm{C}$ overnight. All the primary antibodies were purchased from Cell Signalling (Cell Signalling Technology, Inc). The blots were then washed three times with TBS-T and incubated for $2 \mathrm{~h}$ with HRP-conjugated secondary antibody (Promega, Madison, WI, USA). Immunoreactive bands were developed using an Amersham ECL Plus Western Blotting Detection System (GE Health Care, Buckinghamshire, UK) and visualized by Molecular Imager ${ }^{\circledR}$ VersaDoc ${ }^{\text {TM }}$ MP Imaging Systems (Bio-Rad Laboratories, Hercules, CA, USA).

\section{Statistical analysis}

Statistical analysis was performed using GraphPad Prism software (La Jolla, CA, USA). Data are shown as mean \pm standard error mean, unless otherwise indicated. Group variances were compared using ANOVA and unpaired Student t-test was used to compare means between groups. For all analyses, $P$ value $<0.05$ was considered significant.

\section{Results}

Palmitate induces CCL4 expression in the human monocytic cells

High levels of palmitate and CCL4 have been reported in obesity and T2D. Therefore, we asked if palmitate could induce CCL4 production in human monocytic cells. Our data show that CCL4 mRNA expression levels were significantly higher (THP-1: $77.5 \pm 4.5$ fold; $\mathrm{P}=0.0034$; macrophages: $235.5 \pm 5$ fold, $\mathrm{P}=0.0005$; Primary macrophages: $29.0 \pm 0.5$ fold; $\mathrm{P}=0.0046$ ) in palmitate-treated monocytic cells and macrophages derived from THP-1 cells or from primary monocytes compared to their respective controls (cells treated with vehicle 
only) (Fig. 1A, C \& E). Levels of CCL4 secretory protein were also significantly higher in supernatants from palmitatetreated monocytic cells and macrophages (THP-1 monocytes: $702.1 \pm 19.2 \mathrm{pg} / \mathrm{ml}$, $\mathrm{P}=0.0008$ : macrophages: $493.7 \pm 42.9$ $\mathrm{pg} / \mathrm{ml}, \mathrm{P}=0.009$; primary macrophages: 594.1 $\pm 29.4 ; \mathrm{P}=0.0025$ ) (Fig. 1B, D and F). We further found that palmitate induced CCL4 production was time dependent and reaching its peak between $16-24 \mathrm{hrs}$ and decreasing to low level at 48hrs (Fig. 1G).

\section{TLR4 is involved in palmitate mediated} production of CCL 4

Saturated fatty acids are known to act as agonists for TLR4. Next, we assess if palmitate-induced CCL4 expression in monocytic cells was dependent on TLR4. To address this issue, we used TLR4 receptor blocking antibody and isotype control antibody to label cells before palmitate stimulation. Our data show that anti-TLR4 antibody pre-treatment significantly inhibited the palmitateinduced CCL4 expression at mRNA (Fig. 2A; $\mathrm{P}=0.032$ ) and protein (Fig. 2B; $\mathrm{P}=0.0025)$ levels in THP-1 monocytic cells. Additionally, TLR4 suppression in THP1 cells was also achieved by transfection with TLR4-specific siRNA (2C) and we found that palmitate-induced CCL4 gene/ protein expression was significantly suppressed $(\mathrm{P}<0.05)$ in TLR4 specific siRNA-transfected cells as compared with scramble siRNA-transfected control cells (Fig. 2D and E). We also show that blocking TLR4 ligand-receptor complex internalization does not inhibit the production of CCL4 by palmitate (Fig. 3).

\section{Palmitate mediated CCL4 expression} requires MyD88

MyD88 is a key adaptor protein downstream of TLR-mediated signaling $[13,15]$. Next, we determined whether the palmitate-induced CCL4 expression in THP-1 monocytic cells was MyD88dependent. To address this, we treated THP-1-XBlue ${ }^{\mathrm{TM}}$-defMyD cells (THP-1 cells deficient in MyD88 (MyD88\% THP-1 cells) with palmitate or TNF- $\alpha$ (positive

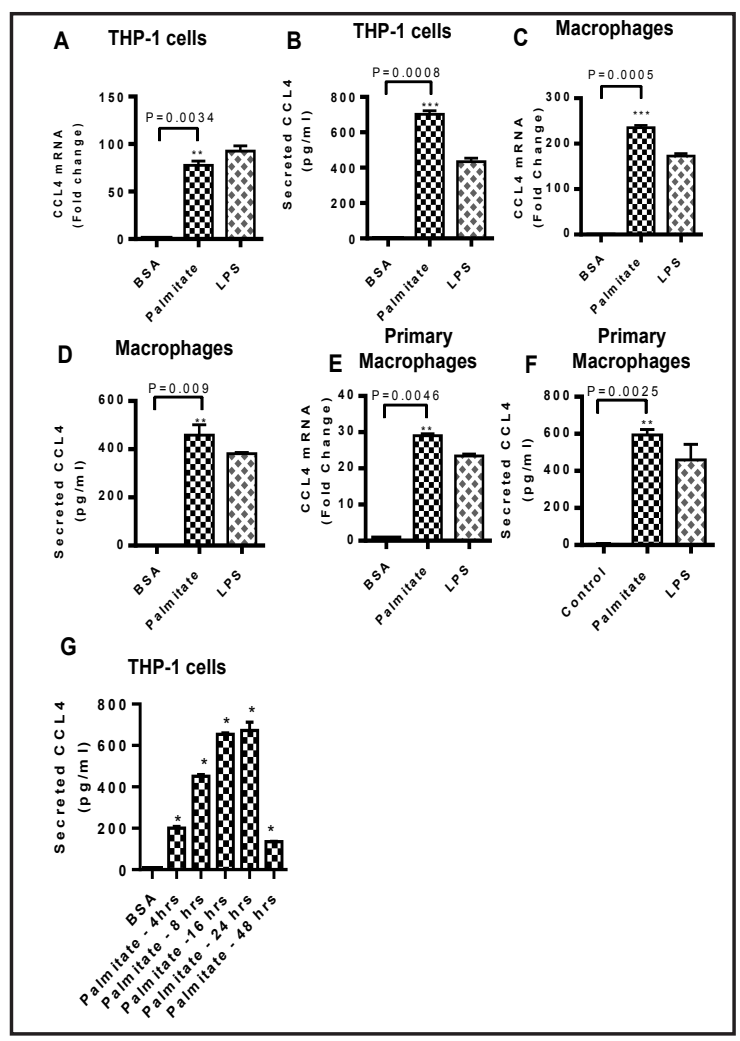

Fig. 1. Effect of palmitate on CCL4 expression in human monocytic cells and macrophages. THP-1 cells were treated with palmitate $(200 \mathrm{uM})$, LPS $(10 \mathrm{ng} / \mathrm{ml}$; positive control) and $0.1 \%$ BSA (vehicle) for $24 \mathrm{hrs}$. Cells and culture media were collected. Total RNA was isolated and CCL4 mRNA was quantified by real time PCR. Relative mRNA expression was described as fold expression over average of gene expression in BSA treated cells (A). Secreted CCL4 protein in culture media was determined by ELISA (B). THP-1 cells were differentiated into macrophages with PMA $(50 \mathrm{ng} / \mathrm{ml})$ for 72 hours and treated with palmitate, LPS and BSA for 24 hrs. Cells and culture media were collected. CCL4 mRNA was determined by real time PCR (C). Secreted CCL4 in culture media was determined by ELISA (D). Monocytes were isolated from PBMCs of healthy volunteers and differentiated into macrophages as described in material and methods. Macrophages were treated with Palmitate, TNF- $\alpha$ and BSA for 24 hrs. Cells and culture media were collected. CCL4 mRNA was determined by real time PCR (E). Secreted CCL4 in culture media was determined by ELISA (F). Cells were treated with palmitate and supernatants were collected at different time points as indicated. CCL4 was determined by ELISA (G). Data are represented as mean \pm SEM. Statistical analysis was done using t-test and One WayANOVA. $\mathrm{P}<0.05$ was considered as statistically significant $* \mathrm{P}<0.01{ }^{* *} \mathrm{P}<0.001,{ }^{* * *} \mathrm{P}<0.0001$. 
Fig. 2. Inhibition of TLR4 down-regulates the palmitate induced CCL4. THP-1 cells were treated with $2 \mu \mathrm{g} /$ $\mathrm{mL}$ of neutralizing TLR4 $\mathrm{mAb}$ or isotype-matched control (IgA) for 40 minutes. Antibody-treated cells were stimulated with palmitate or BSA and incubated for $24 \mathrm{hrs}$. Cells and culture media were collected. Total RNA was isolated from cells and CCL4 mRNA was quantified by real time PCR (A) and secreted CCL4 protein was determined in the culture media by ELISA (B). THP-1 cells were transfected with either control siRNA (30nM) or siRNA (30nM) against TLR4 and incubated $36 \mathrm{hrs}$. Real time PCR was done to measure TLR4 expression (C). Cells were treated with palmitate and BSA for 24 hrs. Real time PCR was done to measure CCL4 mRNA expression (D) and ELISA was done to measure secreted CCL4 protein (E). The data are presented as mean \pm SEM. Statisti-

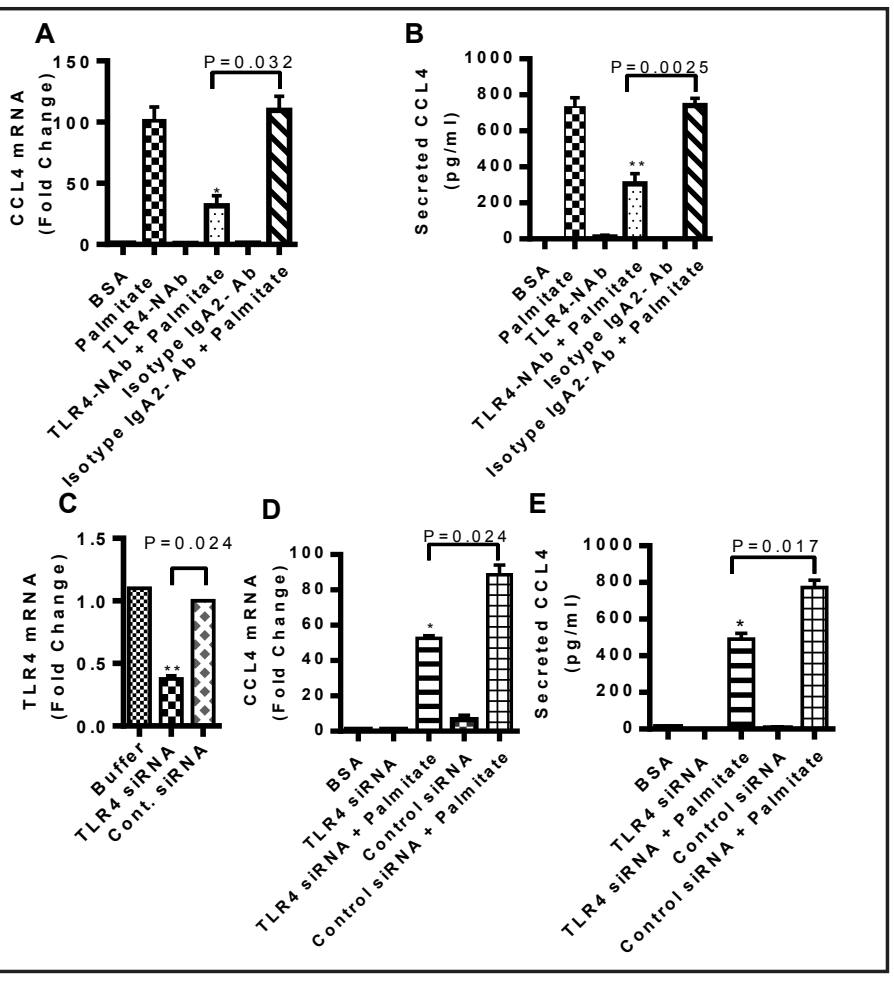
cal analysis was done using t-test and One Way-ANOVA.. $\mathrm{P}<0.05$ was considered as statistically significant $* \mathrm{P}<0.01 ;{ }^{* *} \mathrm{P}<0.001,{ }^{* * *} \mathrm{P}<0.0001$.

Fig. 3. Effect of endocytosis inhibitor on Palmitate induced CCL4. THP-1 cells were pretreated with chloropromazine (an inhibitor of clathrin dependent endocytosis; 10uM) and Methyl- $\beta$-cyclodextrin (an inhibitor of clathrin independent endocytosis; $2 \mathrm{mM}$ ) for $1 \mathrm{hr}$ and then treated with Palmitate for 24 hrs. Supernatants were collected. Cell culture supernatants were assayed for secreted CCL4 protein as determined by ELISA. The results obtained from three independent experiments are shown. The data are presented as mean \pm SEM. Statistical analysis was done using t-test and One Way-ANOVA. $\mathrm{P}<0.05$ was considered as statistically significant $* \mathrm{P}<0.01$; ${ }^{* *} \mathrm{P}<0.001,{ }^{* * *} \mathrm{P}<0.0001$.

control). Our data show that palmitate-induced CCL4 expression was significantly reduced in MyD88\%- THP-1 cells at both mRNA and protein levels (Fig. 4A and B) whereas the TNF- $\alpha$-induced

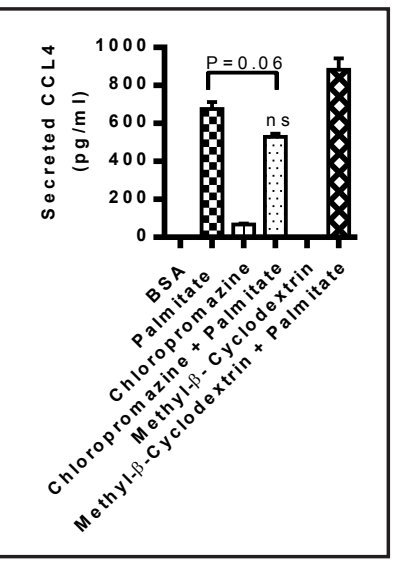
CCL4 expression was not affected in MyD88-/- cells as it was independent of MyD88 pathway (Fig. 4A and B). These data further confirm the TLR4mediated expression of CCL4 in monocytic cells.

MAPK, NF-kB and PI3K/Akt signaling pathways are involved in palmitate induced CCL4 production

MAPK and NF-kB signaling pathways are activated downstream of TLR4. We next asked whether these molecules play role in palmitate induced CCL4 production. Palmitate increased phosphorylation of JNK, MEK/ERK, JNK, c-jun (Fig. 5A). The expression of CCL4 mRNA was reduced (Fig. 5B; P $<0.05$ ) by treatment with inhibitors of either MAPK/JNK (SP600125) or MEK/ERK (PD98059, U0126). Consistent with qRT-PCR results, palmitate induced CCL4 levels were suppressed $(\mathrm{P}<0.05)$ in condition media of THP-1 cells pretreated 


\section{Cellular Physiology Cell Physiol Biochem 2018;46:953-964

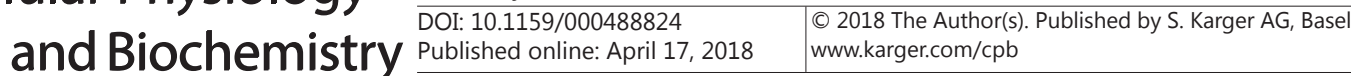 \\ Kochumon et al.: Free Fatty Acid Palmitate Induces CCL4}

Fig. 4. MyD88 deficiency reduced the palmitate induced expression of CCL4. THP-1 XBlue defMyD cells (cells deficient in MyD88 activity) were treated with palmitate (200um) or $0.1 \%$ BSA (Vehicle) or TNF- $\alpha$ (10ng/ml; MyD88 independent stimulus). Cells and culture media were collected after $24 \mathrm{hrs}$. CCL4 gene expression was determined by real time PCR (A) and secreted CCL4 protein was determined in culture media by ELISA (B). The data are presented as mean \pm SEM. Statistical analysis was done using t-test and One Way-ANOVA. $\mathrm{P}<0.05$ was considered as statistically significant ${ }^{*} \mathrm{P}<0.01 ;{ }^{* *} \mathrm{P}<0.001,{ }^{* * *} \mathrm{P}<0.0001$.

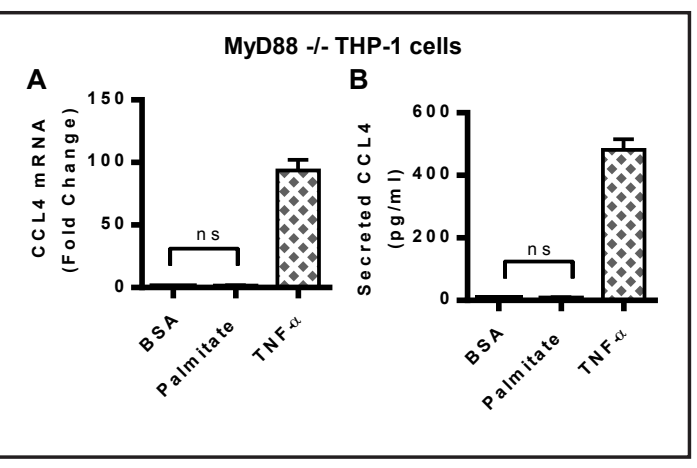

Fig. 5. Palmitate induces phosphorylation of MAPK signaling pathways. THP-1 cells were treated with Palmitate for different time points and cell lysates were prepared as described in methods. Phosphorylated ERK1/2, c-JUN and JNK are depicted in the upper panels and total respective proteins are shown in the lower panels (A). THP-1 cells were pretreated with MEK-ERK inhibitors (U0126: 10uM; PD98059: 10uM) or JNK inhibitor (SP600125: 20uM) for $1 \mathrm{hr}$ and then treated with Palmitate for $24 \mathrm{hrs}$. Cells and supernatants were collected. Cells were used for the isolation of total RNA to assess

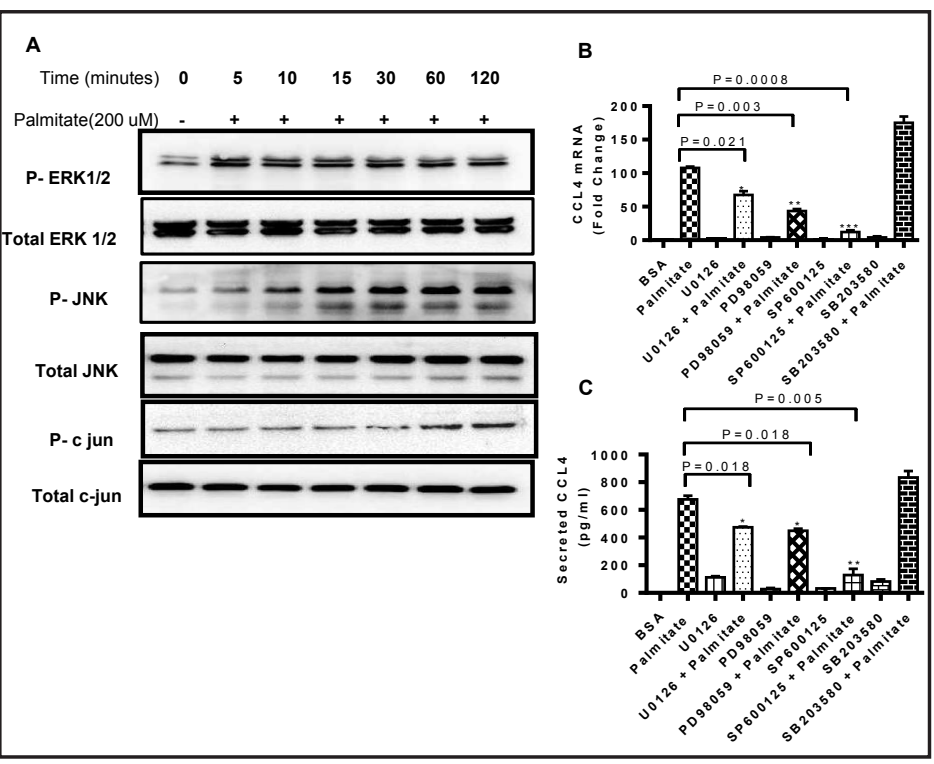
the CCL4 gene expression by realtime RT-PCR (B). Secreted levels of CCL4 protein were determined in supernatants by ELISA (C). The results obtained from three independent experiments are shown. The data are presented as mean \pm SEM. Statistical analysis was done using t-test and One Way-ANOVA. $\mathrm{P}<0.05$ was considered as statistically significant* $\mathrm{P}<0.01 ;{ }^{* *} \mathrm{P}<0.001,{ }^{* * *} \mathrm{P}<0.0001$.

with inhibitors of either MAPKs or MEK/ERK (Fig. 5C). Phosphorylation of NF-kappaB were increased when the cells were treated with palmitate (Fig. 6A). The use of NF- $\kappa B$ inhibitors (BAY 11-7805, Triptolide and Resveratrol), significantly reduced the CCL4 mRNA $(P<0.05)$ and protein expression $(\mathrm{P}<0.05)$ (Fig. 6B \& $\mathrm{C}$ ).

Role of PI3-kinase/Akt in LPS-induced inflammatory responses was reported. Since palmitate activate TLR4 signaling, we first studied the capability of palmitate to activate P13Kinase/Akt. We found that that palmitate induced phosphorylation of P13K in monocytic cells (Fig. 7A). To confirm that P13K/Akt activation is involved in palmitate induced CCL4 production, cells were pretreated with PI 3- kinase inhibitors LY294002 and wortmannin. Both inhibitors suppressed the palmitate induced production of CCL4 (Fig. 7B \& C).

\section{Palmitate CCL4 gene expression requires NF- $k B$ and AP-1 activation}

Previous studies show that CCL4 promoter has binding sites for NF-kB and AP-1[18] . Our data showed that NF-kB and MAPK signaling pathways are activated when cells exposed to palmitate. We next asked if the palmitate mediated CCL4 induction in THP-1 cells was related with the increased NF- $\mathrm{KB} / \mathrm{AP} 1$ activity in these cells. We confirmed the involvement 
Fig. 6. Palmitate activates NF- $\kappa B$ signaling pathways. THP-1 cells were treated with palmitate for different time points and cell lysates were prepared as described in methods. Phosphorylated NF- $\mathrm{BB}$ are depicted in the upper panel and total respective proteins are shown in the lower panel (A). As shown, palmitate treatment increases the phosphorylation of NF- $\kappa B$ in a time-dependent manner. THP-1 cells were pretreated with NF-кB inhibitors (BAY 117085: 10uM; Triptolide: 10uM Resveratrol $15 \mathrm{uM}$ ) for $1 \mathrm{hr}$ and then treated with palmitate for 24 hrs. Cells and supernatants were collected. Cells were used for the isolation of total RNA to assess the CCL4 gene expression by real-time RT-PCR (B). Secreted levels of CCL4 protein were determined in supernatants by ELISA (C). The results

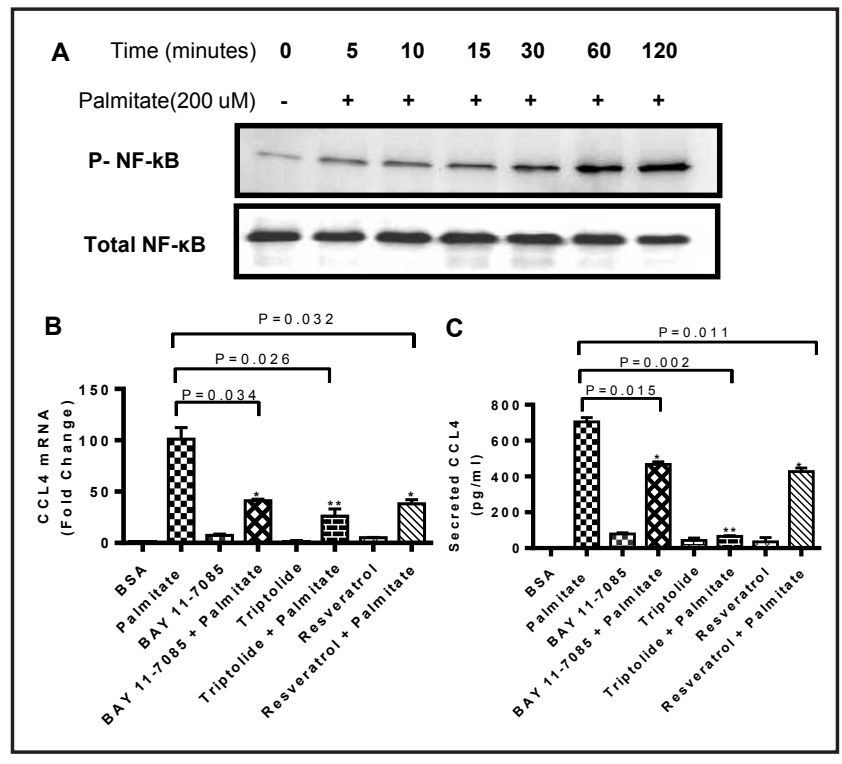
obtained from three independent experiments are shown. The data are presented as mean \pm SEM. Statistical analysis was done using t-test and One Way-ANOVA. $\mathrm{P}<0.05$ was considered as statistically significant * $\mathrm{P}<0.01 ;{ }^{* *} \mathrm{P}<0.001,{ }^{* * *} \mathrm{P}<0.0001$.

Fig. 7. Palmitate activates PI3K signaling pathway during CCL4 production. THP1 cells were treated with palmitate for different time points and cell lysates were prepared as described in methods. Phosphorylated PI3K are depicted in the upper panel and total respective proteins are shown in the lower panel (A). As shown, Palmitate treatment increases the phosphorylation of PI3K in a time-dependent manner. THP-1 cells were pretreated with PI3K Inhibitors (LY294002: 50uM; Wortmannin: $100 \mathrm{nM}$ ) for $1 \mathrm{hr}$ and then treated with Palmitate for 24 hrs. Cells and supernatants were collected. Cells were used for the isolation of total RNA

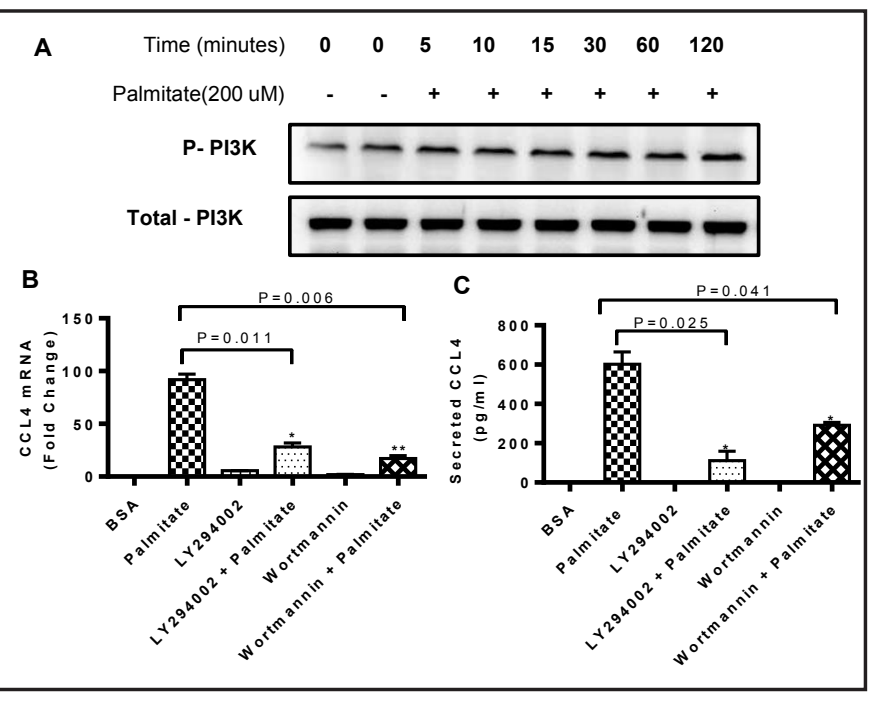
to assess the CCL4 gene expression by real-time RT-PCR (B). Secreted levels of CCL4 protein were determined in supernatants by ELISA (C). The data are presented as mean \pm SEM. Statistical analysis was done using t-test and One Way-ANOVA. P $<0.05$ was considered as statistically significant ${ }^{*} \mathrm{P}<0.01 ;{ }^{* *} \mathrm{P}<0.001,{ }^{* * *} \mathrm{P}<0.0001$.

of NF- $\kappa \mathrm{B} / \mathrm{AP} 1$ in palmitate-induced CCL4 production by using culture media obtained from palmitate-treated THP-1 cells that were expressing a reporter driven by NF- $\kappa \mathrm{B}$ and AP-1 response elements to analyze NF- $\kappa B / A P-1$ activity. As expected, NF- $\kappa B / A P-1$ activity

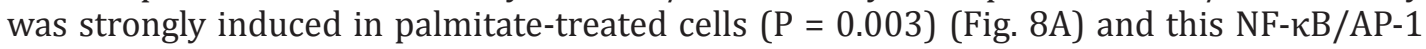
induction correlated with CCL4 secretion (Fig. 8B). In addition, the lack of MyD88 also led to significantly reduced NF- $\mathrm{BB} / \mathrm{AP}-1$ activity and protein following palmitate treatment (Fig. $8 C \& D)$. These data suggest that palmitate-induced upregulation of CCL4 gene expression in monocytic cells involves activation of the NF- $\kappa \mathrm{B} / \mathrm{AP}-1$ transcription factors

\section{KARGER}




\section{Cellular Physiology Cell Physiol Biochem 2018;46:953-964

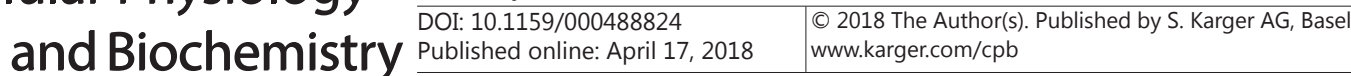 \\ Kochumon et al.: Free Fatty Acid Palmitate Induces CCL4}

Fig. 8. NF- $\kappa \mathrm{B} / \mathrm{AP}-1$ activation is associated with palmitate induced CCL4 production. THP-1-XBlue cells (THP-1 cells stably expressing a secreted embryonic alkaline phosphatase (SEAP) reporter inducible by $\mathrm{NF}-\kappa \mathrm{B}$ and AP-1) were treated with palmitate or BSA or TNF- $\alpha$ for 24 hrs. Culture media were collected. Cell culture media were assayed for SEAP reporter activity (degree of NF- $\kappa \mathrm{B} / \mathrm{AP}-1$ activation) along with CCL4 protein production (A and B). Similarly THP-1$\mathrm{XBlue}^{\mathrm{TM}}$-defMyD cells (Cells deficient in MyD88 activity) were treated with palmitate (200um), TNF- $\alpha$ (10ng/ml) and $0.1 \%$ BSA for 24 hrs. SEAP reporter activity (degree of NF- $\kappa \mathrm{B} / \mathrm{AP}-1$ activation) along with the secreted CCL4 protein was determined in the cell culture media (C and D). The results obtained from three independent experiments are shown. The data are presented as mean \pm SEM. Statistical analysis was done using t-test and One Way-ANOVA. P $<0.05$ was considered as statistically significant $* \mathrm{P}<0.01$; ${ }^{* *} \mathrm{P}<0.001,{ }^{* * *} \mathrm{P}<0.0001$.

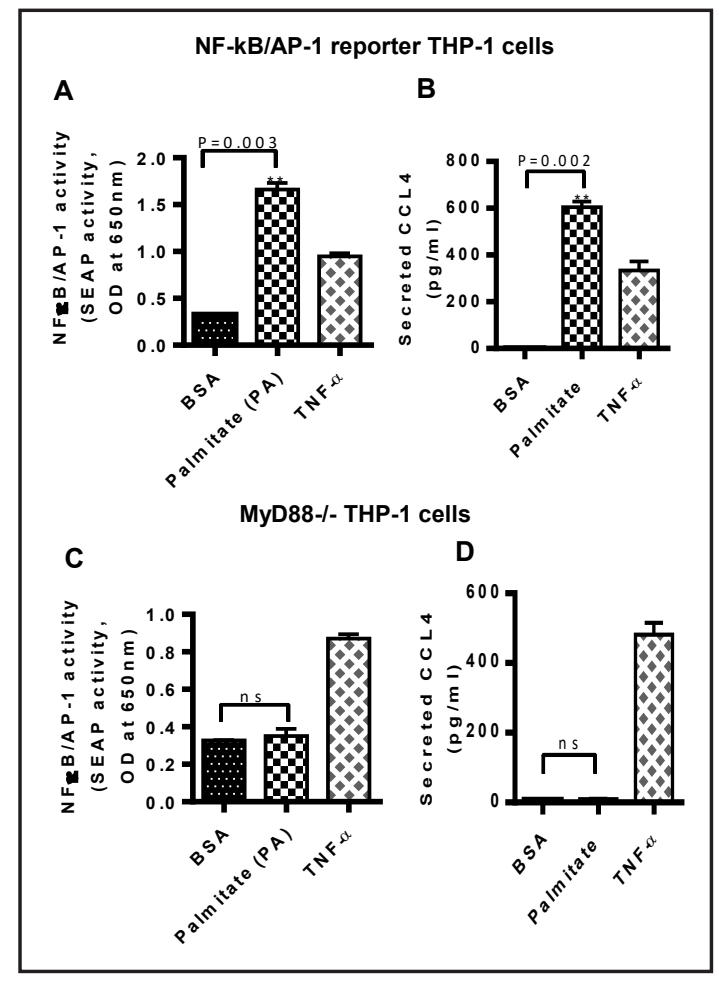

\section{Discussion}

Free fatty acid palmitate and CCL4 are found to be increased in obesity/T2D and are believed to be important components of metabolic inflammation $[8,19,20]$. CCL4 plays a key role in the migration of monocytes into the adipose tissue and thus it contributes to metabolic inflammation [1, 21]. Here, we investigated the production of the CCL4 by free fatty acid palmitate in monocytic cells/macrophages. We show for the first time, to our knowledge, that palmitate which is one of the most abundant FFAs in the human circulation induces expression of CCL4 in monocytic cells. Similarly, palmitate upregulates CCL4 production in macrophages derived either from primary monocytes or THP-1 cells. This indicates that palmitate is one of the novel potent CCL4 inducers in monocytes/macrophages.

We and others showed high expression of TLRs in obese individuals [22, 23]. TLRs are stimulated by SFAs and are involved in metabolic inflammation. TLR4 has been recognized as a main receptor that can be activated by FFAs. SFAs activate TLR4 signaling pathway in macrophages $[10,24,25]$. We and others reported that palmitate-activated TLR4 signaling in monocytes/macrophages results in the production of inflammatory mediators. TLR4 expression on immune cells of monocytic lineage is well known and we found that these cells expressed high levels of CCL4 in response to activation by palmitate. Next, we wanted to assess the role of TLR4-mediated signaling in the production of CCL4 by palmitate-treated monocytic cells and macrophages. Our results showed that the neutralization of TLR4 significantly reduced palmitate-induced CCL4 production by monocytic cells. Furthermore, CCL4 production was also significantly suppressed in monocytic cells that were transfected with TLR4-siRNA. Upon ligand recognition, TLRs recruit TIR domain-containing adaptor proteins such as MyD88 and TRIF, which initiate signal transduction pathways including NF-kB and MAP kinases that lead to the expression of cytokines and chemokines. We also confirmed that MyD88 was required for palmitate mediated CCL4 production.

NF-kB and MAPKs are classical signaling molecules downstream of TLRs' signaling pathways and we also show that palmitate enhances the phosphorylation of ERK 1/2, JNK and NF-kB. Our results further show that the inhibition of MAPKs, specifically ERK1/2 and JNK, significantly blocks the production of CCL4 induced by palmitate. However, p38 
MAPKs involvement was not observed in this induction. ERK 1/2and JNK can be activated by a variety of stimuli in various types of cells [26]. Our findings are consistent with our previous studies showing that activation of MAPKs pathways, except MAPK p38, is important for the production of MMP-9 $[10,14]$. We also identified that inhibition of NF-kB pathways significantly suppresses the production of CCL4. This is also consistent with our previous study which shows that inhibition of NF-kB pathway suppressed the production of MMP9 which is induced by palmitate via TLR4/MyD88 dependent mechanism. TLR4/NF-kB activation is involved in cytokines and chemokines gene expression when cells are stimulated with LPS [27]. In addition, palmitate enhances the activation of NF-kB/AP-1. Activation of NF$\kappa \mathrm{B}$ was, at least in part, responsible for IL-1 $\beta$-mediated CCL4 expression in the hepatocytes [28]. Similarly, we found that NF-kB activation by TLR4 signaling is involved in palmitate mediated CCL4 production. LPS activates AKT kinase and P13kinase in endothelial cells via TLR4/MyD88. AKT kinase is a key downstream target of phosphoinositide 3 kinase (PI3-kinase and PI3-kinase activation is necessary for AKT phosphorylation. Also, MyD88 defective cells did not show AKT kinase activity in response to LPS [29]. Interestingly, our findings show that inhibition of AKT kinase and PI 3-kinase activity suppressed the palmitate induced CCL4 production by monocytes/macrophages. TLR4 mediated cytokine production by macrophages is associated with PI3 kinase activity [30]. Our results suggest that AKT/PI3kinase activity is an important mediator in palmitate TLR4/MyD88 dependent production of CCL4.

\section{Conclusion}

Taken together, our findings suggest that palmitate induces CCL4 expression in monocytes/macrophages via the TLR4/MyD88 dependent activation of NF-kB/MAP Kinases and AKT/PI3 Kinases which may have immunobiological significance with regard to the adipose tissue inflammation in obesity/T2D.

\section{Abbreviations}

AP-1 (Activator protein 1); BSA (Bovine Serum Albumin); ATCC (American Type Culture Collection); CCL3 (Chemokine (C-C motif) ligand 3); CCL4 (Chemokine (C-C motif) ligand 4); ECM (Extracellular matrix); ELISA (Enzyme-linked immunosorbent assay); FFA (Free fatty acid); FSL-1 (Fibroblast-stimulating lipopeptide-1); GAPDH (Glyceraldehyde-3phosphate dehydrogenase); HKLM (Heat killed Listeria monocytogenes); Pam3CSK4 (Pam3Cys-Ser-Lys4); LP (Lipopeptide); LPS (Lipopolysaccharide); LTAs (Lipoteichoic acids); MAPK (Mitogen-activated protein kinase); MBCD (Methyl- $\beta$-cyclodextrin); MMP-9 (Matrix metalloproteinase-9); MyD88 (Myeloid differentiation factor 88); NF- $\kappa B$ (Nuclear factorkappaB); PA (Palmitate (palmitic acid)); PAMPs (Pathogen-associated molecular patterns); PBMC (Peripheral blood mononuclear cells); PVDF (Polyvinylidene difluoride membrane); SFA (Saturated fatty acid); SEAP (Secreted embryonic alkaline phosphatase); Th17 (T helper-17); THP-1 (A human monocytic cell line); TIR (Interleukin (IL)-1 receptor called toll-IL1-R); TLRs (Toll-like receptors); TNF- $\alpha$ (Tumor necrosis factor-alpha); TRAM (TRIFrelated adaptor molecule); TRIF (TIR-domain-containing adapter-inducing IFN- $\beta$ ).

\section{Acknowledgements}

This study was financially supported by Kuwait Foundation for the Advancement of Sciences (KFAS). Grant \# RC14016001 (RA-AM-2017-007).

SK performed experiments and participated in writing the paper. AW, BC, and SS participated in performing experiments. Sindhu S participated in writing paper. JT critically 


\section{Cellular Physiology Cell Physiol Biochem 2018;46:953-964

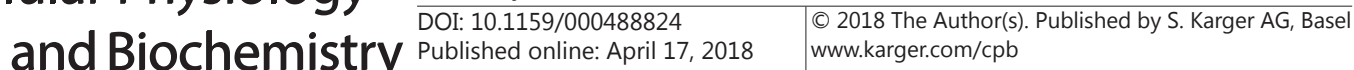

Kochumon et al.: Free Fatty Acid Palmitate Induces CCL4

reviewed and commented the manuscript. RA conceived the idea, supervised research, designed experiments, analyzed the data, and wrote the manuscript.

\section{Disclosure Statement}

The authors declare that they have no competing interests.

\section{References}

1 von Stebut E, Metz M, Milon G, Knop J, Maurer M: Early macrophage influx to sites of cutaneous granuloma formation is dependent on mip-1alpha /beta released from neutrophils recruited by mast cell-derived tnfalpha. Blood 2003;101:210-215.

- Maurer M, von Stebut E: Macrophage inflammatory protein-1. Int J Biochem Cell Biol 2004;36:1882-1886.

-3 Dai YJ, Li YY, Zeng HM, Liang XA, Xie ZJ, Zheng ZA, Pan QH, Xing YX: Effect of pharmacological intervention on mip-1alpha, mip-1beta and mcp-1 expression in patients with psoriasis vulgaris. Asian Pac J Trop Med 2014;7:582-584.

4 Barczyk A, Pierzchala E, Caramori G, Sozanska E: Increased expression of ccl4/mip-1beta in cd8+ cells and cd4+ cells in sarcoidosis. Int J Immunopathol Pharmacol 2014;27:185-193.

5 Mrugacz M: Ccl4/mip-1beta levels in tear fluid and serum of patients with cystic fibrosis. J Interferon Cytokine Res 2010;30:509-512.

6 Boven LA, Montagne L, Nottet HS, De Groot CJ: Macrophage inflammatory protein-1alpha (mip-1alpha), mip-1beta, and rantes mrna semiquantification and protein expression in active demyelinating multiple sclerosis (ms) lesions. Clin Exp Immunol 2000;122:257-263.

7 Vila LM, Molina MJ, Mayor AM, Cruz JJ, Rios-Olivares E, Rios Z: Association of serum mip-1alpha, mip1 beta, and rantes with clinical manifestations, disease activity, and damage accrual in systemic lupus erythematosus. Clin Rheumatol 2007;26:718-722.

-8 Mathews JA, Wurmbrand AP, Ribeiro L, Neto FL, Shore SA: Induction of il-17a precedes development of airway hyperresponsiveness during diet-induced obesity and correlates with complement factor d. Front Immunol 2014;5:440.

-9 Boden G: Obesity and free fatty acids. Endocrinol Metabol Clinic North Am 2008;37:635-646, viii-ix.

10 Sindhu S, Al-Roub A, Koshy M, Thomas R, Ahmad R: Palmitate-induced mmp-9 expression in the human monocytic cells is mediated through the tlr4-myd88 dependent mechanism. Cell Physiol Biochem 2016;39:889-900.

11 Jialal I, Kaur H, Devaraj S: Toll-like receptor status in obesity and metabolic syndrome: A translational perspective. J Clin Endocrinol Metab 2014;99:39-48.

12 Caesar R, Tremaroli V, Kovatcheva-Datchary P, Cani PD, Backhed F: Crosstalk between gut microbiota and dietary lipids aggravates wat inflammation through tlr signaling. Cell Metab 2015;22:658-668.

13 Ahmad R, El Bassam S, Cordeiro P, Menezes J: Requirement of tlr2-mediated signaling for the induction of il-15 gene expression in human monocytic cells by hsv-1. Blood 2008;112:2360-2368.

-14 Al-Rashed F, Kochumon S, Usmani S, Sindhu S, Ahmad R: Pam3csk4 induces mmp-9 expression in human monocytic thp-1 cells. Cell Physiol Biochem 2017;41:1993-2003.

-15 Ahmad R, Shihab PK, Jasem S, Behbehani K: Fsl-1 induces mmp-9 production through tlr-2 and nf-kappab / ap-1 signaling pathways in monocytic thp-1 cells. Cell Physiol Biochem 2014;34:929-942.

16 Haversen L, Danielsson KN, Fogelstrand L, Wiklund O: Induction of proinflammatory cytokines by longchain saturated fatty acids in human macrophages. Atherosclerosis 2009;202:382-393.

17 Staiger H, Staiger K, Stefan N, Wahl HG, Machicao F, Kellerer M, Haring HU: Palmitate-induced interleukin-6 expression in human coronary artery endothelial cells. Diabetes 2004;53:3209-3216.

18 Gonsalves C, Kalra VK: Endothelin-1-induced macrophage inflammatory protein-1beta expression in monocytic cells involves hypoxia-inducible factor-1alpha and ap- 1 and is negatively regulated by microrna-195 J Immunol 2010;185:6253-6264. 


\section{Cellular Physiology Cell Physiol Biochem 2018;46:953-964

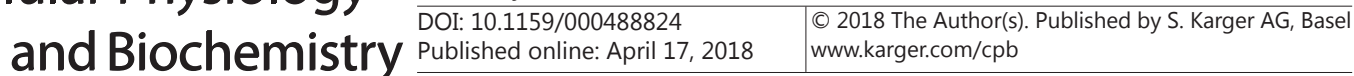

Kochumon et al.: Free Fatty Acid Palmitate Induces CCL4

-19 Dahlman I, Kaaman M, Olsson T, Tan GD, Bickerton AS, Wahlen K, Andersson J, Nordstrom EA, Blomqvist L, Sjogren A, Forsgren M, Attersand A, Arner P: A unique role of monocyte chemoattractant protein 1 among chemokines in adipose tissue of obese subjects. J Clin Endocrinol Metab 2005;90:5834-5840.

20 Hardy OT, Perugini RA, Nicoloro SM, Gallagher-Dorval K, Puri V, Straubhaar J, Czech MP: Body mass indexindependent inflammation in omental adipose tissue associated with insulin resistance in morbid obesity. Surg Obes Relat Dis 2011;7:60-67.

-21 Surmi BK, Hasty AH: The role of chemokines in recruitment of immune cells to the artery wall and adipose tissue. Vascul Pharmacol 2010;52:27-36.

-22 Schaffler A, Scholmerich J: Innate immunity and adipose tissue biology. Trends Immunol 2010;31:228-235.

-23 Ahmad R, Al-Mass A, Atizado V, Al-Hubail A, Al-Ghimlas F, Al-Arouj M, Bennakhi A, Dermime S, Behbehani K: Elevated expression of the toll like receptors 2 and 4 in obese individuals: Its significance for obesityinduced inflammation. J Inflamm (Lond) 2012;9:48.

24 Erridge C, Samani NJ: Saturated fatty acids do not directly stimulate toll-like receptor signaling. Arterioscler Thromb Vasc Biol 2009;29:1944-1949.

25 Huang S, Rutkowsky JM, Snodgrass RG, Ono-Moore KD, Schneider DA, Newman JW, Adams SH, Hwang DH: Saturated fatty acids activate tlr-mediated proinflammatory signaling pathways. J Lipid Res 2012;53:20022013.

26 Firestein GS, Manning AM: Signal transduction and transcription factors in rheumatic disease. Arthritis Rheum 1999;42:609-621.

27 Guijarro-Munoz I, Compte M, Alvarez-Cienfuegos A, Alvarez-Vallina L, Sanz L: Lipopolysaccharide activates toll-like receptor 4 (tlr4)-mediated nf-kappab signaling pathway and proinflammatory response in human pericytes. J Biol Chem 2014;289:2457-2468.

28 Zhang T, Guo CJ, Li Y, Douglas SD, Qi XX, Song L, Ho WZ: Interleukin-1beta induces macrophage inflammatory protein-1 beta expression in human hepatocytes. Cell Immunol 2003;226:45-53.

29 Li X, Tupper JC, Bannerman DD, Winn RK, Rhodes CJ, Harlan JM: Phosphoinositide 3 kinase mediates tolllike receptor 4-induced activation of nf-kappa b in endothelial cells. Infect Immun 2003;71:4414-4420.

30 Ojaniemi M, Glumoff V, Harju K, Liljeroos M, Vuori K, Hallman M: Phosphatidylinositol 3-kinase is involved in toll-like receptor 4-mediated cytokine expression in mouse macrophages. Eur J Immunol 2003;33:597605. 